\title{
Using proteomics as a powerful tool to develop a vaccine against Mediterranean visceral leishmaniasis
}

\author{
Sajad Rashidi ${ }^{1} \cdot$ Kurosh Kalantar $^{2} \cdot$ Gholamreza Hatam $^{3}$
}

Received: 11 December 2017/Accepted: 12 March 2018/Published online: 19 March 2018

(C) Indian Society for Parasitology 2018

\begin{abstract}
Visceral leishmaniasis (VL) is a tropical infectious disease, which is called Mediterranean visceral leishmaniasis (MVL) in the Mediterranean area. In spite of many attempts, no effective commercial vaccine exists for MVL. To find new targets for developing antileishmanial vaccines, knowing parasite antigens that provoke the immune system are on demand. Nowadays, proteomics methods are defined as approaches for analysis of protein profiling of different cells. Within this framework, detection of new antigens is becoming more facilitated. In this review, we aimed to introduce possible targets using proteomics so; they could be used as candidates for developing vaccines against MVL. It can shed new light in the near future on the development of promising vaccines for MVL.
\end{abstract}

Keywords Mediterranean visceral leishmaniasis .

Proteomics - Vaccine target

\section{Introduction}

Visceral leishmaniasis (VL) is caused by Leishmania donovani and L. infantum in the old world, while it is due to $L$. chagasi in the new world. In Mediterranean regions, $L$. infantum is the main cause of Mediterranean visceral

Gholamreza Hatam

hatamghr@sums.ac.ir

1 Department of Parasitology and Mycology, Shiraz University of Medical Sciences, Shiraz, Iran

2 Department of Immunology, Shiraz University of Medical Sciences, Shiraz, Iran

3 Basic Sciences in Infectious Diseases Research Center, Shiraz University of Medical Sciences, Shiraz, Iran leishmaniasis (MVL) (Azizi et al. 2006; Barati et al. 2015; Fakhar et al. 2006; Gharekhani et al. 2016; Ghatee et al. 2013; Hatam et al. 2010). Approximately every year 500,000 new cases of VL occur in the world (Barati et al. 2015; Ghatee et al. 2013). In the zoonotic pattern, reservoirs are dogs and rodents (Postigo 2010; Reithinger et al. 2007; Sabzevari et al. 2013). Evidence has shown that dogs can act as a natural host for MVL, and human is its accidental host (Fig. 1). In VL, parasites tend to infect macrophages throughout the viscera. Manifestations of VL in human are different and vary and sometimes it could appear in a life-threatening progressive visceral form of the disease. For instance, the confection of HIV with VL is a serious problem for health care conditions (Alvar et al. 2008).

With respect to the diagnostic of $\mathrm{VL}$, the methods are different. They include parasitology, biochemical, serological and molecular approaches (Mohammadi-Ghalehbin et al. 2011; Oryan et al. 2013; Rassi et al. 2007). Chemotherapy is the first choice for VL treatment but unfortunately, using the available drugs is accompanied by the side effects (Murray et al. 2005). Side effects and resistance to available drugs prompt the investigators to invent new drugs. Doubtless, an effective vaccine would be an appropriate treatment.

Nowadays, new techniques such as proteomics give us a promising approach for discovering new targets to develop a protective vaccine for infectious diseases like leishmaniasis. The aim of this review was to provide a view about those markers characterized by proteomics technique in previous articles. 


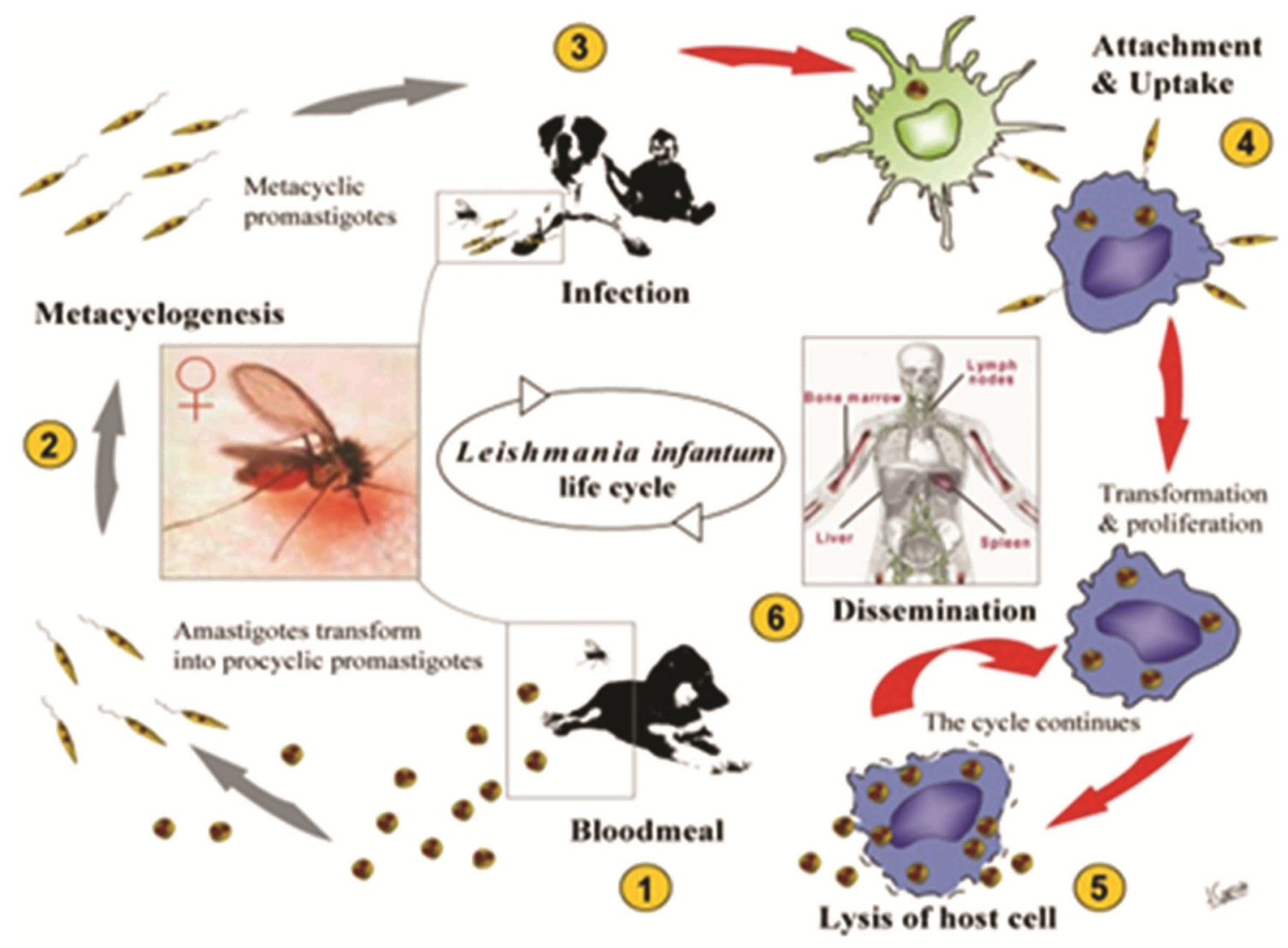

Fig. 1 Life-cycle of L. infantum: Leishmania has a digenetic lifecycle consisting of an extracellular flagellated stage (promastigote) in the sandfly vectors, and an aflagellated intracellular stage (amastigote)

\section{Vaccines for leishmaniasis}

Th1 is responsible for the immunity against the Leishmania. This subset of T- cells by producing of IFN- $\gamma$ can activate the macrophages to kill the parasite (Mansueto et al. 2007; Nylén and Gautam 2010). In the treated patients, it has been shown that recovery and resistance to reinfection are related to the development of antigenspecific Th1 cell responses (Nylen and Gautam 2010; Roberts 2005; Tripathi et al. 2007). Taken together, a good strategy for developing a vaccine against leishmaniasis, such as MVL, should focus on eliciting the Th1 cells against the Leishmania parasites.

Some research groups characterized a number of Leishmania proteins which produced variable protection against Leishmania in animal models (Coler et al. 2007; ForoughiParvar et al. 2015; Foroughi-Parvar and Hatam 2014; Kushawaha et al. 2011; Singh et al. 2012). Also, using killed Leishmania vaccine has shown different results in different studies (Kedzierski 2010; Srivastava et al. 2016). On the other hand, live attenuated ones have shown an effective protection in some studies (Bhattacharya et al. 2015; Gannavaram et al. 2014; Ismail et al. 2017). The use of in the animal reservoirs and human. Reproduced with permission from Nieto et al. (2011)

attenuated Leishmania parasites as a vaccine is very interesting because they are somehow mimicking the natural infection. In addition, it leads to similar immune responses. By means of molecular techniques, the recombinant proteins are becoming another new approach for vaccines development (Coler et al. 2007; Dias et al. 2017). In contrast to the substantial effort to develop a vaccine, there is no approved vaccine against human leishmaniasis, while there are approved vaccines for dogs (Reithinger et al. 2007).

Nowadays, by completion of genome sequencing of Leishmania, it seems that invention of a vaccine is becoming an easier task than before (Cantacessi et al. 2015; Llanes et al. 2015). Recently, researchers have attempted to use proteomics techniques for introducing immunodominant antigens in Leishmania parasites for designing new effective vaccines.

\section{Proteomics}

Proteome refers to the set of proteins encoded by the genome and defined as an analysis of proteins in order to determine their unique identity, quantity, function, and 
interaction (Herosimczyk et al. 2006). Evidence has been shown that this method also practically suitable for analysis of the proteome of Leishmania genus (Murray et al. 2005; Reithinger et al. 2007). As most of the genes are conserved among the Leishmania species, there is a poor correlation between the transcripts and the proteins expressed by the Leishmania parasite (Iantorno et al. 2017) in Leishmania spp. Functional genomic analysis of mRNA also does not show the whole pattern of protein expression. A widespread technique, like western blotting, just looks at the change in one protein, but if we intend to check the changes in a large number of proteins in various biological materials and microorganism at the same time, proteomics facilitates this task (Lewandowicz et al. 2009) and helps us to discover biomarkers which can be applied to drug, vaccine, and diagnostic targets (Thongboonkerd 2004).

\section{Methods and steps involved in proteomics analysis}

Combination of proteomics, bioinformatics and mass spectrometry (MS) could give us a good quality of proteome maps. 2-dimensional gel electrophoresis (2DE) is a method which can separate the proteins mixtures based on the net charge by isoelectric focusing (IEF) (1 dimension) and based on to the molecular weight by sodium dodecyl sulfate-polyacrylamide gel electrophoresis (SDS-PAGE) (2 dimensions). After staining the gels, protein spots were digested by trypsin; then we extracted peptides sequence using tandem mass spectrometry (MS/MS) (Fig. 2) which is linked to a genome sequence database.

For reducing the complexity of proteins, fractionation by ammonium sulfate and digitonin prefractionation apply for detecting the markers with low molecular mass (McNicoll et al. 2006). For enhancing the visualization of targets with low molecular masson the gels, liquid phase IEF should be combined with 2DE (Brobey and Soong 2007).

Recently, proteomics methods have been improved by using multi-dimensional liquid chromatography (LC) and applied for proteomics of pathogenic organisms (Zhang et al. 2010).

\section{Proposed targets for MVL vaccines using proteomics in promastigotes and amastigotes}

To find new targets for developing anti-leishmanial vaccines, knowing the biology of the parasite and exact mechanism of host-parasite interactions are two important issues that are worth noticing. Within this framework, different studies have found out proteins which are involved in parasite's vitality, infectivity, and invasiveness

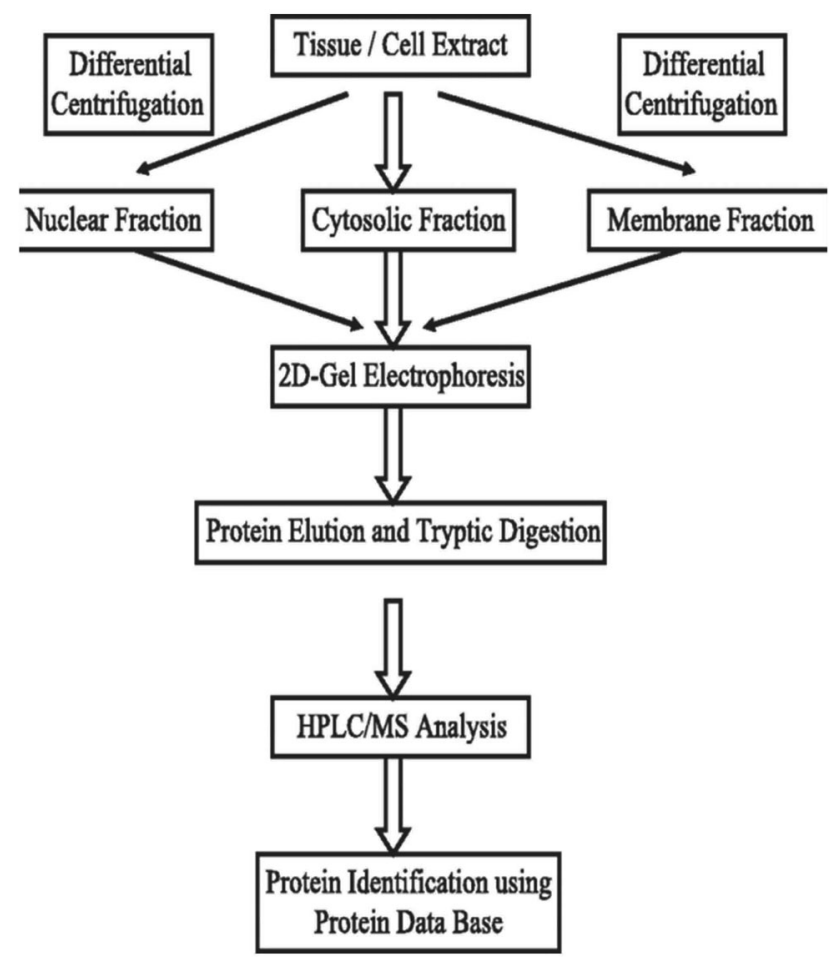

Fig. 2 Overview of steps involved in proteomics analysis: whole cell lysate or a special cell fraction can be analyzed by 2DE and mass spectrometry. Reproduced with permission from Smita et al. (2015)

(da Fonseca et al. 2014; Dea-Ayuela et al. 2006). Expressed proteins responsible for survival and infectivity are not directly related to the vaccine potential targets except the proteins which are immunodominant that can be applied for a vaccine against MVL in future. Recognition of immunodominant proteins by immunoproteomics method seems to be on demand for designing a practical vaccine in MVL.

The results of a study indicated that the immunodominant proteins in $L$. infantum promastigote membranes were located in molecular weight of 30-36 kDa range (KamounEssghaier et al. 2005). The spots sequences showed LACK (Leishmania homolog of receptors for activated C-kinase) and a possible member of the aldehyde reductase family provokes humoral immunity in MVL (Gómez-Arreaza et al. 2011). These proteins belong to the conserved proteins which are permanently expressed in the eukaryotic cells.

Another study, using multiplex 2D on attenuated $L$. infantum promastigotes (Aravind et al. 2003), showed observable changes in the thiol-redox control system and made it less virulent (Daneshvar et al. 2012). This metabolic system is vital and protects the Leishmania against the oxidative burst of macrophages (Acestor et al. 2006). Also, the metabolism of hydrogen peroxide and trypanothione reductase activity in the attenuated type was 
declined in comparison to the wild-type. Consequently, using immunodominant targets of attenuated promastigotes forms is probably helpful in improving vaccines.

A recent study on $L$. infantum promastigote in logarithmic phase introduced some immunodominant proteins such as ATPase beta subunit (chaperone function); propionyl carboxylase (in fatty acid metabolism); transketolase (in carbohydrate metabolism); succinyl-diaminopimelate desuccinylase (in amino acid metabolism and synthesis); proteasome subunit (in protein synthesis and catabolism); full size heat shock protein 70 (in stress response/chaperone) and adrenodoxin reductase, a non-antigenic protein which is related to the ergosterol biosynthesis pathway (Dea-Ayuela et al. 2006). Most of these proteins are immunodominant and have been proved associated with crucial physiological and virulence functions of Leishmania parasite. Thus, these results help us to identify new targets for developing vaccines for $\mathrm{VL}$.

The result of a $2 \mathrm{D}$ immunoblotting study on the latelogarithmic phase of L. infantum promastigotes (Agallou et al. 2016) identified immunodominant proteins mostly include in stress responses and metabolic systems. These data also showed that eukaryotic initiation factor 4a (elF4a) and LACK are known for their immunostimulatory potential and provoke antigen-presenting cells to produce IL-12 and TNF $\alpha$.

Also, in this study, an in silico investigation proposed that the chaperonin HSP60, enolase, cyclophilin 2, dihydrolipoamide dehydrogenase, and cyclophilin 40 are restricted to MHC-I and/or MHC-II. The identification of proteins which are restricted to MHC-I and MHC-II is very crucial to have a long-lasting response of $\mathrm{CD} 8+$ and $\mathrm{CD} 4+$ respectively against the parasites. Based on the finding of this study, apparently, the combination of bioinformatics and immunoproteomics will probably guide us to predict vaccine targets.

The results of an immunoproteomics study on $L$. infantum promastigote stage (Coelho et al. 2012) show that the phosphoglycan beta-1, 3-galactosyltransferase, and flagellum transition zone component, which is linked to LPG synthesis, are more highly expressed. In addition, elongation factors, heat shock proteins such as HSP70, HSP83 and other chaperones, as well as tubulin and other housekeeping proteins, were observed. It seems that HSPs have important roles in immunity. Also, there are targets for the responses of the immunity system to a wide difference of pathogens including fungi, bacteria, helminths, and protozoa (Tsan and Gao 2009). These data are important for designing vaccines and biology of $L$. infantum.

In Leishmania parasites, over-expression of some genes is directly or indirectly related to the infectivity (Alcolea et al. 2009, 2010). In 2011 (Alcolea et al. 2011), an investigation by 2DE and MS processed the proteins of $L$. infantum promastigote in early logarithmic to stationary phase in the replicate axenic cultures. The observation indicated that the level of protein and mRNA in eukaryotic elongation factor 1a (eEF1a) subunit and the electron transfer flavoprotein (ETF), had the equal expression level and in the stationary phase it was decreased. In promastigotes in dividing logarithmic stage, a $51 \mathrm{kDa}$ subunit of replication factor A showed an up-regulation. All of the proteins ascribed here display the same differential regulation values with the identical mRNA levels. Therefore, during the life cycle of $L$. infantum, the $40 \mathrm{~S}$ ribosomal protein S12, the eEF1a subunit, the ETF, a-tubulin and the T-complex protein 1 subunit $\gamma$ are regulated in different patterns both in proteome and transcriptome. It seems that they get close to the target for a vaccine for each stage.

A recent study on L. infantum promastigote in logarithmic phase (Dea-Ayuela et al. 2006) introduced some structural immunodominant targets, such as paraflagellar rod protein 3 (PAR3), which can be effective for vaccine targets in MVL. It has been demonstrated that some structural proteins in Leishmania are functional and several of them are crucial for developmental stages and parasite life cycle. Thus, these results support us to identify new targets for developing vaccines.

The results of a study (Kamoun-Essghaier et al. 2005) illustrated that elongation factor $1 \mathrm{a}$ is a mitochondrial protein which could stimulate the antibody in visceral leishmaniasis. Also, elongation factor 2 and elongation factor 1a are able to induce cellular immunity in leishmaniasis patients (Jaiswal et al. 2014; Kushawaha et al. 2011). All the mentioned proteins in the current investigation belong to the conserved proteins which permanently expressed in eukaryotic cells and could be a possible target for diagnosis and vaccine against MVL.

The results of another study by free flow electrophoresis (FFE) and IEF separation technique (Brotherton et al. 2012; Nissum and Foucher 2008) showed that glycolytic enzymes and flagella proteins upregulated in the $L$. infantum promastigotes. In amastigote, enzymes included in fatty acid $\beta$ oxidation and gluconeogenesis was overexposed. In accordance with this data, post-translational modification occurs in each stage. Consequently, these observations could open a new horizon for developing a new vaccine.

In another research by labeling the cysteine-reactive light and heavy ICAT (Isotope Coded Affinity Tag) reagents on $L$. infantum, (Leifso et al. 2007), the authors displayed the paraflagellar rod proteins (PFR) (Portman and Gull 2010) and cell surface protease leishmanolysin (GP63) (Isnard et al. 2012; Lieke et al. 2008). The PFR is a multifunctional complex of the cytoskeleton structure and the motility of Leishmania parasite is dependent on it 
(Carrilloa et al. 2008). GP63, which is a surface protease in Leishmania, is very important in the virulence and infectivity. Organisms that express gp63 can utilize the opsonic effects of complement while avoiding its lytic effects (Gupta et al. 2013). Thus, we can use these specific proteins to design effective vaccines in future.

The membranous proteins are crucial in the regulatory pathways of Leishmania parasites and host-pathogen interactions. A quantitative mass spectrometry study (Lynn et al. 2013) identified that approximately $20-40 \%$ of proteins in the amastigote and promastigote of $L$. infantum were different in expression. Drawing a map for proteins such as leishmanolysin (GP63), eEF-1 $\alpha$ and amastin based on their functions in the metabolism, infectivity, and virulence could help us to detect proteins which can potentially be candid for inventing the MVL vaccines.

The analysis of proteins in developmental stages of $L$. infantum showed that $6.1 \%$ of proteins were related to the promastigote, and $12.4 \%$ belonged to the amastigotes (McNicoll et al. 2006). The correlation between amastigote specific protein isoforms and its mRNA was almost 53\%, while in respect to the promastigote specific spots, no correlation was observed. A lot of proteins were indicated in multiple spots; consequently, that post-translational modification is too much in this parasite. In some cases, different isoforms seem to be proprietary to different life stages. These data proposed that post-transcriptional controls at translational and post-translational levels could perform remark function in the differentiation of Leishmania. Consequently, this data indicates the necessity of attention to post-translational modification of protein for development of a vaccine for leishmaniasis.

In a proteomics study (Leifso et al. 2007), histone glycolytic proteins and enzyme enolase (Gupta et al. 2014) were introduced in protein profiling of L. infantum. In addition to apoptosis roles, glycolytic proteins are essential for transcriptional control of histone gene expression (Sirover 2005). Based on the essential role of these proteins, they can be probable targets in VL vaccines in future.

In a proteomics study, the authors checked the virulence factors in the amastigote forms extracted from the macrophages (in vitro) and hamster (in vitro) tissues (da Fonseca et al. 2014). The results illustrated that over-expression of KMP-11, phosphomannomutase, metallopeptidase, EF-2, Rieske iron-sulfur protein precursor, and $S$-adenosylhomocysteine is associated to the virulence and degradation of host cell protective proteins. Also, tryparedoxin peroxidase and peroxiredoxin keep the $L$. infantum safe against the stress conditions (Iyer et al. 2008). On the other hand, EF-1 $\alpha$ inhibits the activation of macrophage and chaperones, and endoribonuclease L-PSP has a role in the prolongation of host cell lifetime and parasite survival. In conclusion, these proteins are indispensable for the parasite survival and pathogenesis; thus, targeting of these significant proteins should be considered for focusing on MVL vaccines.

In an immunoproteomics study on antigenic extracts of L. infantum axenic amastigotes (Coelho et al. 2012), ATPdependent RNA helicase (Barhoumi et al. 2006) and amastin (Nasereddin et al. 2010) were presented. These proteins are comparable to the tissue amastigotes. In addition, elongation factors, heat shock proteins such as HSP70, HSP83, and other chaperones, as well as tubulin and other housekeeping proteins, have been observed. These data are in the same line with those of other studies (Moreira and Murta 2016; Ramírez et al. 2013). In addition, to use these markers in the improvement of vaccines for MVL, by focusing on these proteins, we can reach more information about the biology of $L$. infantum. Thus, further studies are recommended to be done in this regard.

An investigation in Canada (El Fakhry et al. 2002) used comparative $2 \mathrm{DE}$ and $\mathrm{MS}$ to introduce proteins that are differentially presented in the amastigote of $L$. infantum. They identified two proteins, which belong to the isocitrate dehydrogenase, energetic metabolism pathways, and the glycolytic enzyme triosephosphate isomerase. Isocitrate dehydrogenase exists in the tricarboxylic acid cycle, a metabolic pathway in which acetate is oxidized to produce ATP. Triosephosphate isomerase is the other protein indicating a high presentation in amastigotes, which is a high current enzyme that roles as an essential duty in glycolysis (Kushawaha et al. 2012). The kinetic analysis illustrates that their activity and amount are more in amastigote in comparison to promastigote. For instance, Triosephosphate isomerase (TIM) activity in L. infantum amastigotes was twofold higher in comparison to L. infantum promastigotes, probably due to amastigotes need to high levels of TIM activity to make ATP via glycolysis within host cells (Bente et al. 2003). Generally, these enzymes have a crucial role in metabolism, survival, and pathogenicity of the amastigote in L. infantum so can be effective in designing vaccines for MVL.

\section{Proposed targets for MVL vaccines using proteomics in the excretory-secretory antigens (ES) of L. infantum promastigotes}

Nowadays, scientists believe that excretory-secretory antigens (ES) are appropriate sources for immune system stimulating and vaccines in MVL (Joshi et al. 2014; Lemesre et al. 2005; Petitdidier et al. 2016). Shotgun proteomics approaches revealed that the proteins in secretions of $L$. infantum promastigote are associated with the nucleotide metabolism, carbohydrate metabolism, antioxidant activity, protein degradation, heat-shock response, and 
other processes. In this survey, the ES immunodominant proteins were checked with the sera of animals with clinical manifestation for canine visceral leishmaniasis (CVL). In addition to the new research avenues in MVL probable vaccines (Braga et al. 2014), these isolated proteins are important to check the immune responses and the pathology of L. infantum.

The results of another immunoproteomics study on soluble exogenous antigens (SEAgs) of $L$. donovani $(\mathrm{Ku}-$ mar et al. 2015) indicated that enolase, carboxypeptidase and activated protein kinase $\mathrm{C}$ receptor homolog deviate the immune responses to the Th1 cells; consequently, they are suitable targets for vaccine development. Also, heatshock 70-related protein1, glucose-regulated protein 78 and heat-shock $70-\mathrm{kDa}$ proteins have been introduced as the inducer of Th1 immune responses. The finding of these new targets may be our dream in case of VL vaccines comes true. Since the $L$. donovani and $L$. infantum are two agents of VL in different areas, we are convinced to focus on the results of this research to propose these targets as a vaccine for MVL in the future.

\section{Proteomics and drug resistance and the targets that can be proposed for MVL vaccine}

In a study, the mitochondrion of L. infantum was exposed to Antimony (SbIII) and Miltefosine (MIL) (Vincent et al. 2015). Although these drugs induce the cell death through the changes on the Leishmania mitochondrion, the parasite can be adapted and withstand these drugs by changes in the proteins' pattern. Generally, mitochondrial DNA adapts the parasite to the different nutritional condition and also helps the parasite to grow in different hosts. That is why; the results of these studies can guide us to target the special proteins in the mitochondrion. Thus, by evaluation of their immunogenicity, we can reach a new source of antigens for designing a vaccine against Leishmania spp. Also, Hide et al. (2008) studied the mitochondrial protein content of $L$. infantum, using proteomics. They present mitochondrial fraction with lowest levels of cytosolic contamination by performing fractionation with the focus and enrichment of mitochondrial proteins.

A comparative proteomics study on resistance and sensitive strains of $L$. infantum to the Amphotericin B (AmB) (Brotherton et al. 2014) showed that in the resistance mutant strain, up-regulation was seen in the proteins which belonged to the glycolysis and tricarboxylic acid cycle. Interestingly, up-regulation was observed in reactive oxygen species (ROS) scavenging and heat shock proteins in the resistant mutant. Knowing the protein profiles of sensitive and resistant strains of $L$. infantum can improve our information about the selection of appropriate targets for MVL vaccines.

\section{Conclusion}

As reviewed above, proteomics technique gives us a facility for identification of protein profiling of Leishmania parasites. After identification of protein targets, we should identify immunodominant proteins which provoke the immune responses against $L$. infantum. These introduced targets can be evaluated in Elisa, western blotting and in vivo to prove their immunostimulatory property for designing a vaccine.

It seems that most of the proteins that reviewed the candidates for VL vaccines are located in the metabolism pathway of Leishmania parasites. Additionally, some of these proteins are located in the other part of the parasite such as flagella. It proposes that for obtaining more appropriate and effective vaccine targets, 2DE and mass spectrometry should be performed on the special fraction of the parasite, not on the whole parasite lysate.

Acknowledgements This article was supported by Shiraz University of Medical Sciences under the Grant Number 94-7597.

Author contributions Sajad Rashidi: Data collection and manuscript writing; Kurosh kalantar: Project development, manuscript editing, and data analysis; Hatam Gholamreza: Project development, manuscript editing.

\section{Compliance with ethical standards}

Conflict of interest The authors declare that they have no conflict of interests.

\section{References}

Acestor N, Masina S, Ives A, Walker J, Saravia NG, Fasel N (2006) Resistance to oxidative stress is associated with metastasis in mucocutaneous leishmaniasis. J Infect Dis 194:1160-1167

Agallou M, Athanasiou E, Samiotaki M, Panayotou G, Karagouni E (2016) Identification of immunoreactive Leishmania infantum protein antigens to asymptomatic dog sera through combined immunoproteomics and bioinformatics analysis. PLoS ONE 11:e0149894

Alcolea PJ, Alonso A, Larraga V (2009) Genome-wide analysis reveals increased levels of transcripts related with infectivity in peanut lectin non-agglutinated promastigotes of Leishmania infantum. Genomics 93:551-564

Alcolea PJ, Alonso A, Gómez MJ, Moreno I, Domínguez M, Parro V et al (2010) Transcriptomics throughout the life cycle of Leishmania infantum: high down-regulation rate in the amastigote stage. Int J Parasitol 40:1497-1516

Alcolea PJ, Alonso A, Larraga V (2011) Proteome profiling of Leishmania infantum promastigotes. J Eukaryot Microbiol 58:352-358 
Alvar J, Aparicio P, Aseffa A, Den Boer M, Canavate C, Dedet JP et al (2008) The relationship between leishmaniasis and AIDS: the second 10 years. Clin Microbiol Rev 21:334-359

Aravind L, Iyer LM, Anantharaman V (2003) The two faces of Alba: the evolutionary connection between proteins participating in chromatin structure and RNA metabolism. Genome Biol 4:R64

Azizi K, Rassi Y, Javadian E, Motazedian M, Rafizadeh S, Yaghoobi Ershadi M et al (2006) Phlebotomus (Paraphlebotomus) alexandri: a probable vector of Leishmania infantum in Iran. Ann Trop Med Parasitol 100:63-68

Barati M, Mohebali M, Alimohammadian MH, Khamesipour A, Akhoundi B, Zarei Z (2015) Canine visceral leishmaniasis: seroprevalence survey of asymptomatic dogs in an endemic area of northwestern Iran. J Parasit Dis 39:221-224

Barhoumi M, Tanner N, Banroques J, Linder P, Guizani I (2006) Leishmania infantum LeIF protein is an ATP-dependent RNA helicase and an eIF4A-like factor that inhibits translation in yeast. FEBS J 273:5086-5100

Bente M, Harder S, Wiesgigl M, Heukeshoven J, Gelhaus C, Krause $E$ et al (2003) Developmentally induced changes of the proteome in the protozoan parasite Leishmania donovani. Proteomics 3:1811-1829

Bhattacharya P, Dey R, Dagur PK, Kruhlak M, Ismail N, Debrabant A et al (2015) Genetically modified live attenuated Leishmania donovani parasites induce innate immunity through classical activation of macrophages that direct the Th1 response in mice. Infect Immun 83:3800-3815

Braga MS, Neves LX, Campos JM, Roatt BM, de Oliveira Aguiar Soares RD et al (2014) Shotgun proteomics to unravel the complexity of the Leishmania infantum exoproteome and the relative abundance of its constituents. Mol Biochem Parasitol 195:43-53

Brobey RK, Soong L (2007) Establishing a liquid-phase IEF in combination with 2-DE for the analysis of Leishmania proteins. Proteomics 7:116-120

Brotherton M-C, Racine G, Ouameur AA, Leprohon P, Papadopoulou B, Ouellette M (2012) Analysis of membrane-enriched and high molecular weight proteins in Leishmania infantum promastigotes and axenic amastigotes. J Proteome Res 11:3974-3985

Brotherton M-C, Bourassa S, Légaré D, Poirier GG, Droit A, Ouellette M (2014) Quantitative proteomic analysis of amphotericin B resistance in Leishmania infantum. Int J Parasitol Drugs Drug Resist 4:126-132

Cantacessi C, Dantas-Torres F, Nolan MJ, Otranto D (2015) The past, present, and future of Leishmania genomics and transcriptomics. Trends Parasitol 31:100-108

Carrilloa E, Crusat M, Nietoa J, Chicharroa C, Martinezc E, Valladares B et al (2008) Immunogenicity of HSP-70, KMP11 and PFR-2 leishmanial antigens in the experimental model of canine visceral leishmaniasis. Vaccine 26:1902-1911

Coelho VT, Oliveira JS, Valadares DG, Chávez-Fumagalli MA, Duarte MC, Lage PS et al (2012) Identification of proteins in promastigote and amastigote-like Leishmania using an immunoproteomic approach. PLoS Negl Trop Dis 6:e1430

Coler RN, Goto Y, Bogatzki L, Raman V, Reed SG (2007) Leish111f, a recombinant polyprotein vaccine that protects against visceral Leishmaniasis by elicitation of CD4 $+\mathrm{T}$ cells. Infect Immun 75:4648-4654

da Fonseca Pires S, Fialho LC Jr, Silva SO, Melo MN, de Souza CC, Tafuri WL et al (2014) Identification of virulence factors in Leishmania infantum strains by a proteomic approach. J Proteome Res 13:1860-1872

Daneshvar H, Wyllie S, Phillips S, Hagan P, Burchmore R (2012) Comparative proteomics profiling of a gentamicin-attenuated Leishmania infantum cell line identifies key changes in parasite thiol-redox metabolism. J Proteom 75:1463-1471
Dea-Ayuela MA, Rama-Iñiguez S, Bolás-Fernández F (2006) Proteomic analysis of antigens from Leishmania infantum promastigotes. Proteomics 6:4187-4194

Dias DS, Ribeiro PAF, Martins VT, Lage DP, Ramos FF, Dias ALT et al (2017) Recombinant prohibitin protein of Leishmania infantum acts as a vaccine candidate and diagnostic marker against visceral leishmaniasis. Cell Immunol. https://doi.org/10.1016/j.cellimm.2017.11.001

El Fakhry Y, Ouellette M, Papadopoulou B (2002) A proteomic approach to identify developmentally regulated proteins in Leishmania infantum. Proteomics 2:1007-1017

Fakhar M, Asgari Q, Motazedian M, Mohebali M, Hatam G, Alborzi A (2006) First report of Kala-azar from Qeshm Island in Persian Gulf. Iran J Parasitol 1:53-56

Foroughi-Parvar F, Hatam G (2014) Vaccines for canine leishmaniasis advances in preventive medicine. Adv Prev Med. https://doi.org/10.1155/2014/569193

Foroughi-Parvar F, Hatam G-R, Sarkari B, Kamali-Sarvestani E (2015) Leishmania infantum FML pulsed-dendritic cells induce a protective immune response in murine visceral leishmaniasis. Immunotherapy 7(1):3-12. https://doi.org/10.2217/imt.14.102

Gannavaram S, Dey R, Avishek K, Selvapandiyan A, Salotra P, Nakhasi HL (2014) Biomarkers of safety and immune protection for genetically modified live attenuated Leishmania vaccines against visceral leishmaniasis-discovery and implications. Front Immunol 5:241

Gharekhani J, Heidari H, Hajian-Bidar H, Abbasi-Doulatshahi E, Edalati-Shokat H (2016) Prevalence of anti-Leishmania infantum antibodies in dogs from West of Iran. J Parasit Dis 40:964-967

Ghatee MA, Sharifi I, Haghdoost AA, Kanannejad Z, Taabody Z, Hatam G et al (2013) Spatial correlations of population and ecological factors with distribution of visceral leishmaniasis cases in southwestern Iran. J Vector Borne Dis 50:179

Gómez-Arreaza A, Acosta H, Barros-Álvarez X, Concepción JL, Albericio F, Avilan L (2011) Leishmania mexicana: LACK (Leishmania homolog of receptors for activated C-kinase) is a plasminogen binding protein. Exp Parasitol 127:752-761

Gupta G, Oghumu S, Satoskar AR (2013) Mechanisms of immune evasion in leishmaniasis. Adv Appl Microbiol 82:155-184. https://doi.org/10.1016/B978-0-12-407679-2.00005-3

Gupta R, Kumar V, Kushawaha PK, Tripathi ChP, Joshi S, Sahasrabuddhe AA et al (2014) Characterization of glycolytic enzymes-rAldolase and rEnolase of Leishmania donovani, identified as Th1 stimulatory proteins, for their immunogenicity and immunoprophylactic efficacies against experimental visceral leishmaniasis. PLoS ONE 9(1):e86073. https://doi.org/10.1371/journal.pone.0086073

Hatam GR, Adnani SJ, Asgari Q, Fallah E, Motazedian MH, Sadjjadi SM et al (2010) First report of natural infection in cats with Leishmania infantum in Iran. Vector Borne Zoonotic Dis 10:313-316

Herosimczyk A, Dejeans N, Sayd T, Ozgo M, Skrzypczak W, Mazur A (2006) Plasma proteome analysis: 2D geld and chips. J Physiol Pharmacol 57:81

Hide M, Ritleng A-S, Brizard J-P, Monte-Allegre A, Sereno D (2008) Leishmania infantum: tuning digitonin fractionation for comparative proteomic of the mitochondrial protein content. Parasitol Res 103:989-992

Iantorno SA, Durrant C, Khan A, Sanders MJ, Beverley SM, Warren WC et al (2017) Gene expression in Leishmania is regulated predominantly by gene dosage. MBio. https://doi.org/10.1128/mbio.01393-17

Ismail N, Kaul A, Bhattacharya P, Gannavaram S, Nakhasi HL (2017) Immunization with live attenuated Leishmania donovani Centrin $^{-1-}$ parasites is efficacious in asymptomatic infection. Front Immunol 8:1788 
Isnard A, Shio MT, Olivier M (2012) Impact of Leishmania metalloprotease GP63 on macrophage signaling. Front Cell Infect Microbiol 2:72

Iyer JP, Kaprakkaden A, Choudhary ML, Shaha C (2008) Crucial role of cytosolic tryparedoxin peroxidase in Leishmania donovani survival, drug response and virulence. Mol Microbiol 68:372-391

Jaiswal A, Khare P, Joshi S, Kushawaha P, Sundar S (2014) Th1 stimulatory proteins of Leishmania donovani: comparative cellular and protective responses of rTriose phosphate isomerase, rProtein disulfide isomerase and rElongation factor- 2 in combination with rHSP70 against visceral leishmaniasis. PLoS ONE 9(9):e108556. https://doi.org/10.1371/journal.pone.0108556

Joshi S, Rawat K, Yadav NK, Kumar V, Siddiqi MI, Dube A (2014) Visceral leishmaniasis: advancements in vaccine development via classical and molecular approaches. Front Immunol 5:380

Kamoun-Essghaier S, Guizani I, Strub JM, Van Dorsselaer A, Mabrouk K, Ouelhazi L et al (2005) Proteomic approach for characterization of immunodominant membrane-associated 30-to 36-kilodalton fraction antigens of Leishmania infantum promastigotes, reacting with sera from mediterranean visceral leishmaniasis patients. Clin Diagn Lab Immunol 12(2):310-320. https://doi.org/10.1128/cdli.12.2.310-320.2005

Kedzierski L (2010) Leishmaniasis vaccine: where are we today? J Glob Infect Dis 2:177

Kumar A, Samant M, Misra P, Khare P, Sundar S, Garg R et al (2015) Immunostimulatory potential and proteome profiling of Leishmania donovani soluble exogenous antigens. Parasite Immunol 37:368-375

Kushawaha PK, Gupta R, Sundar S, Sahasrabuddhe AA, Dube A (2011) Elongation factor-2, a Th1 stimulatory protein of Leishmania donovani, generates strong IFN- $\gamma$ and IL-12 response in cured Leishmania-infected patients/hamsters and protects hamsters against Leishmania challenge. J Immunol 187:6417-6427

Kushawaha PK, Gupta R, Tripathi CDP, Khare P, Jaiswal AK, Sundar $S$ et al (2012) Leishmania donovani triose phosphate isomerase: a potential vaccine target against visceral leishmaniasis. PLoS ONE 7(9):e45766. https://doi.org/10.1371/journal.pone.0045766

Leifso K, Cohen-Freue G, Dogra N, Murray A, McMaster WR (2007) Genomic and proteomic expression analysis of Leishmania promastigote and amastigote life stages: the Leishmania genome is constitutively expressed. Mol Biochem Parasitol 152:35-46

Lemesre J-L, Holzmuller P, Cavaleyra M, Gonçalves RB, Hottin G, Papierok G (2005) Protection against experimental visceral leishmaniasis infection in dogs immunized with purified excreted secreted antigens of Leishmania infantum promastigotes. Vaccine 23:2825-2840

Lewandowicz A, Bakun M, Imiela J, Dadlez M, Specjalistyczny MS, Mas ŚLS (2009) Proteomics in uronephrology new perspectives of noninvasive diagnostics? Nefrol Dial Pol 13:15-21

Lieke T, Nylén S, Eidsmo L, McMaster WR, Mohammadi AM, Khamesipour A et al (2008) Leishmania surface protein gp63 binds directly to human natural killer cells and inhibits proliferation. Clin Exp Immunol 153:221-230

Llanes A, Restrepo CM, Del Vecchio G, Anguizola FJ, Lleonart R (2015) The genome of Leishmania panamensis: insights into genomics of the L. (Viannia) subgenus. Sci Rep 5:8550. https://doi.org/10.1038/srep08550

Lynn MA, Marr AK, McMaster WR (2013) Differential quantitative proteomic profiling of Leishmania infantum and Leishmania mexicana density gradient separated membranous fractions. J Proteom 82:179-192

Mansueto P, Vitale G, Di Lorenzo G, Rini G, Mansueto S, Cillari E (2007) Immunopathology of leishmaniasis: an update. Int J Immunopathol Pharmacol 20:435-445
McNicoll F, Drummelsmith J, Müller M, Madore É, Boilard N, Ouellette $\mathrm{M}$ et al (2006) A combined proteomic and transcriptomic approach to the study of stage differentiation in Leishmania infantum. Proteomics 6:3567-3581

Mohammadi-Ghalehbin B, Hatam GR, Sarkari B, Mohebali M, Zarei Z, Jaberipour $\mathrm{M}$ et al (2011) A Leishmania infantum FMLELISA for the detection of symptomatic and asymptomatic canine visceral leishmaniasis in an endemic area of Iran. Iran J Immunol 8:244

Moreira DS, Murta SM (2016) Involvement of nucleoside diphosphate kinase $\mathrm{b}$ and elongation factor 2 in Leishmania braziliensis antimony resistance phenotype. Parasit Vectors 9:641

Murray HW, Berman JD, Davies CR, Saravia NG (2005) Advances in leishmaniasis. The Lancet 366:1561-1577

Nasereddin A, Schweynoch C, Schonian G, Jaffe CL (2010) Characterization of Leishmania (Leishmania) tropica axenic amastigotes. Acta Trop 113:72-79

Nieto A, Domínguez-Bernal G, Orden JA, De La Fuente R, MadridElena N, Carrión J (2011) Mechanisms of resistance and susceptibility to experimental visceral leishmaniosis: BALB/c mouse versus syrian hamster model. Vet Res 42:39

Nissum M, Foucher AL (2008) Analysis of human plasma proteins: a focus on sample collection and separation using free-flow electrophoresis. Expert Rev Proteomics 5:571-587

Nylen S, Gautam S (2010) Immunological perspectives of leishmaniasis. J Glob Infect Dis 2:135

Nylén S, Gautam S (2010) Immunological perspectives of leishmaniasis. J Glob Infect Dis 2:135

Oryan A, Shirian S, Tabandeh MR, Hatam GR, Kalantari M, Daneshbod Y (2013) Molecular, cytological, and immunocytochemical study and kDNA sequencing of laryngeal Leishmania infantum infection. Parasitol Res 112:1799-1804

Petitdidier E, Pagniez J, Papierok G, Vincendeau P, Lemesre J-L, BrasGonçalves R (2016) Recombinant forms of Leishmania amazonensis excreted/secreted promastigote surface antigen (PSA) induce protective immune responses in dogs. PLoS Negl Trop Dis 10(5):e0004614. https://doi.org/10.1371/journal.pntd.0004614

Portman N, Gull K (2010) The paraflagellar rod of kinetoplastid parasites: from structure to components and function. Int $\mathbf{J}$ Parasitol 40:135-148

Postigo JAR (2010) Leishmaniasis in the world health organization eastern mediterranean region. Int J Antimicrob Agents 36:S62S65

Ramírez CA, Requena JM, Puerta CJ (2013) Alpha tubulin genes from Leishmania braziliensis: genomic organization, gene structure and insights on their expression. BMC Genom 14:454

Rassi Y, Azizi K, Motazedian M, Javadian E, Rafizadeh S, Fakhar M et al (2007) The seminested PCR based detection of Leishmania infantum infection in asymptomatic dogs in a new endemic focus of visceral leishmaniasis in Iran. J Arthropod Borne Dis 1:38-42

Reithinger R, Dujardin J-C, Louzir H, Pirmez C, Alexander B, Brooker S (2007) Cutaneous leishmaniasis. Lancet Infect Dis 7:581-596

Roberts M (2005) Current understandings on the immunology of leishmaniasis and recent developments in prevention and treatment. Br Med Bull 75:115-130

Sabzevari S, Razmi GR, Naghibi A, Khoshnegah J (2013) A serological study of Leishmania infantum in dogs of Khorasan Razavi province, Iran. J Parasit Dis 37:189-191

Singh OP, Stober CB, Singh AK, Blackwell JM, Sundar S (2012) Cytokine responses to novel antigens in an Indian population living in an area endemic for visceral leishmaniasis. PLoS Negl Trop Dis 6(10):e1874. https://doi.org/10.1371/journal.pntd.0001874

Sirover MA (2005) New nuclear functions of the glycolytic protein, glyceraldehyde-3-phosphate dehydrogenase, in mammalian cells. J Cell Biochem 95:45-52 
Smita K, Qadar Pasha MA, Jain SK (2015) High altitude illness and adaptation: hints from proteomics. J Proteomics Bioinform S3:004. https://doi.org/10.4172/jpb.S3-004

Srivastava S, Shankar P, Mishra J, Singh S (2016) Possibilities and challenges for developing a successful vaccine for leishmaniasis. Parasit Vectors 9:277

Thongboonkerd V (2004) Proteomics in nephrology: current status and future directions. Am J Nephrol 24:360-378

Tripathi P, Singh V, Naik S (2007) Immune response to Leishmania: paradox rather than paradigm. FEMS Microbiol Rev 51:229-242
Tsan MF, Gao B (2009) Heat shock proteins and immune system. J Leukoc Biol 85:905-910

Vincent IM, Racine G, Légaré D, Ouellette M (2015) Mitochondrial proteomics of antimony and miltefosine resistant Leishmania infantum. Proteomes 3:328-346

Zhang X, Fang A, Riley CP, Wang M, Regnier FE, Buck C (2010) Multi-dimensional liquid chromatography in proteomics-a review. Anal Chim Acta 664(2):101-113. https://doi.org/10.1016/j.aca.2010.02.001 